\title{
Transcriptome analysis of blastocysts acquiring implantation competency in mice
}

\author{
S Kong ${ }^{1,2}$ and $\mathrm{H}$ Wang $^{1,2}$ \\ ${ }^{1}$ Reproductive Medical Center, The First Affiliated Hospital of Xiamen University, Xiamen, \\ Fujian, People's Republic of China \\ ${ }^{2}$ School of Medicine Xiamen University, Xiamen, Fujian, People's Republic of China
}

Corresponding author email: shuangbo kong@163.com

\begin{abstract}
Blastocyst activation, referring to the blastocyst acquiring the implantation competency, is the determining factor for implantation into the receptive uterus. It involves the process of embryonic cell differentiation to contact and initiate dialog with the uterine cells. Before the application of -omics approaches to this biological event, only the cellular morphological changes and a small number of molecules were known to regulate this process. This review aims to discuss the recent exploration for global molecular changes during the blastocyst activation in mouse model, based on a transcriptional evaluation strategy.
\end{abstract}

\section{Introduction}

The embryo development in the womb is a main characteristic of mammalian species, and the embryo first becomes embedded into the endometrium, a process known as the embryo implantation, for the further in vivo development in the uterus. Prior to implantation, the embryo develops into the blastocyst stage with two distinct cell lineages, the outer specialized trophectoderm (TE) and the inner cell mass (ICM) [1].

Implantation only occurs within a limited time duration, defined as the window of implantation [2]. During this window, the uterus is in the receptive state, which is mainly under the coordinated regulation of ovary-produced steroid hormones progesterone (P4) and estrogen (E2) both in humans and mice $[3,4]$. The duration of window of implantation is usually at 7-9 days after the LH surge and consequent ovulation in human menstrual cycle and on day 4 of pregnancy (day $1=$ vaginal plug) in mice [5]. The embryo, a blastocyst in this stage, also undergoes extensive molecular remodeling to achieve implantation competency and to initiate the crosstalk with the uterus [2].

In mice, dynamic hormonal changes occur during the pre-implantation stage and dominate the corresponding differentiation of uterine cells, including the epithelium and stroma. On day 1 of pregnancy, the uterine epithelium undergoes extensive cell proliferation, due to the stimulatory effect of a preovulatory E2 surge. From day 3 onward, as the newly formed corpus luteum begins to produce $\mathrm{P} 4$, epithelial proliferation is inhibited, and stromal cell proliferation is initiated. On day 4 morning, the epithelium ceases proliferation and stromal cells undergo an extensive proliferation. Thereafter, the pre-implantation ovarian E2 surge on day 4 morning acts as the critical trigger for occurrence of implantation. Ovariectomy on day 4 morning, before this pre-implantation E2 surge, induces delayed implantation, a state in which the blastocyst in the uterine cavity that cannot implant into the uterus. This state can be maintained by daily P4 injection from day 5 and 
terminated by the administration of E2 $[6,7]$. During delayed implantation, the blastocyst is in a state of dormancy without implantation competency, and the uterus is in the neutral phase.

By employing this experimentally-induced delayed implantation model and embryo transfer, it was demonstrated that, not only is uterine receptivity critical for implantation, but also the state of the blastocyst determines the implantation window in the receptive uterus [7]. Utilizing the embryo transfer model, it was demonstrated that the implantation window is shorter for dormant blastocysts than for normal or activated blastocysts after E2 activation in delayed model. Later, it was found that E2 regulates the uterus entering into the receptive state through the nuclear receptor $E R \alpha$, and the $E 2$ metabolite, catechol 4-hydroxy-E2, a compound rapidly produced by uterine cell after the E2 supplement, target the embryo directly to allow the blastocyst acquiring the implantation competency in the delayed model [8].

While a wide range of regulatory molecules have been identified as essential players in conferring uterine receptivity in both laboratory animal models and humans $[2,9]$, it remains largely unknown how blastocysts achieve implantation competency both in normal and delayed implantation in mice. From this perspective, the delayed implantation model permits addressing the issue of the molecular mechanisms underlying implantation competency of the blastocyst, as the dormant blastocyst shows almost all characteristics of the normal blastocyst except for implantation competency and metabolic activity. Moreover, deep understanding of the difference between the dormant and activated blastocyst will provide some clue to how animal cells can be maintained in a low metabolic and dormant state for an extended period and still have the potential to rapidly recover from this dormancy after a physiological stimulation. Utilizing the delayed implantation model to acquire blastocysts with different implantation competence, we and others have conducted-omics studies of the RNA and protein levels to explore the global molecular difference during the blastocyst activation [10-12]. For our RNA-seq experiment, we collected the dormant (implantation incompetent), reactivating ( 6 hours post E2 treatment), and reactivated (12 hours post E2 treatment) blastocysts to explore the differentially expressed genes as the blastocyst acquires implantation competency. In this review, we discuss our recent findings based on the transcriptome data, combined with related studies in the delayed mouse model.

\section{Metabolism and cell proliferation activity}

During delayed implantation, the embryo suspends its development at the blastocyst stage, entering a dormancy state showing low cellular activity, such as energy metabolism, cell proliferation, and translation [13]. Consistently, the differentially expressed genes in our RNA-Seq data reveal that the most significantly enriched signal pathways are related to metabolic processes and cell proliferation during blastocyst activation. Specifically, the genes involved in the biosynthetic processes, including the lipid synthesis, protein synthesis, and nucleic acid synthesis are increased, whereas the catabolic processes are consistently down-regulated. Moreover, cell proliferation resumes from the low activity in the dormant state, as revealed by ki67-positive signaling, principally in the ICM and polar TE cells of the activated blastocyst. This enhanced cell proliferation activity, appears mainly due to the enhanced expression of positive regulators, such as cyclin E family members along with the decreased expression of negative regulators, such as Cdkn1a (p21) and Cdkn1b (p27) during the activation process [12]. The increased biomass synthesis also facilitates the resumption of cell proliferation from the dormant state. During the process of activation from the dormancy, it was also found that the genes related to the glycolysis, tricarboxylic acid cycle, respiratory electron transport, and ATP synthesis are up-regulated significantly in the reactivated blastocyst, which is consistent with increased ATP production in the activated blastocyst [11]. 
The mTOR and myc signaling pathways have been shown to be critical during embryo dormancy, since their inhibition can induce the normal blastocyst to enter into a biosynthetic dormancy state $[14,15]$, mimicking the physiological in vivo dormant blastocyst. The RNA-Seq data also showed negative regulation of these pathways, such as Akt1s1/Tsc1/Fnip1 in mTOR signaling during diapause. Mnt/Mxd4 in Myc signaling, are highly expressed in the dormant blastocyst, and once the blastocyst has been activated by the estrogen supplement, these negative regulators are sharply downregulated, accompanied by the activation of mTOR and myc signaling. This information gives us more clues toward understanding the modulation of myc and mTOR activity in physiological situation, at least at the transcriptional level. Autophagy-related genes, which are negatively regulated by the mTOR signaling pathway, also displayed down-regulation during blastocyst activation, which is consistent with the previous report that the autophagy is indispensable for the maintain the dormant state $[16,17]$. Polyamines are important chemical compound essential for both male and female reproductive processes and the embryo/fetal development [18]. Fenelon et al. reported that the inhibition of uterine ODC1, the rate limiting enzyme of polyamine synthesis, induced the uterus entering into a quiescence state that causes entry of the blastocyst into diapause [19]. Our data also suggest that, during the delayed implantation model, the polyamines produced by embryos are inhibited, as the negative regulator for polyamine synthesis Oaz1 was highly expressed in the dormant blastocyst. The molecular target for the polyamines produced during the blastocyst activation in delayed model should be further investigated, as it was reported that polyamines can regulated RNA translation through hypusination modification of translation related factor eIF5A [20].

\section{Cellular stemness vs differentiation of different cell lineages in the blastocyst}

Blastocyst dormancy is a suspension of developmental program, with the blastocyst floating in the uterine cavity for a certain period of time without the progression into the post-implantation development. The first embryonic stem cell line was derived from the inner cell mass cell of the dormant blastocyst, suggesting that the ICM cells maintained the pluripotency or cell stemness during the blastocyst dormancy [21]. Comparison of RNA-seq data of dormant and activated blastocyst with that of the normal developmental program of day 4 and day 4.5 ICM cells [22] shows that the embryo development-related genes displayed similar tendency of expression changes, implying that the ICM differentiation program is conserved in both on-time and delayed implantation. One prominent biological event during peri-implantation is X-chromosome reactivation in the epiblast of female blastocyst ICM cells, which will undergo random inactivation during post-implantation development [23]. Accompanying X-chromosome reactivation, the Xist, an X-chromosome transcribed long noncoding RNA which inactivates the X-chromosome in cis, is downregulated. During blastocyst activation, Xist is significantly downregulated, with upregulation of other X-chromosome linked genes, which are reported to be actively transcribed at a later stage [22]. These observations suggest $X$-chromosome reactivation in female blastocyst has been initiated, but fails to be completed, even though the dormant blastocyst has survived for several days.

Trophectoderm is the surface component the blastocyst directly contacts the uterine epithelium and mediates the invasion of embryo into the endometrial bed. The trophectoderm located nearest to the ICM is designated polar TE, and opposite farthest from ICM, mural TE. During the attachment of embryo to the uterus in mice, mural TE cells are the main contact with the uterine epithelium in mice, which is different from the situation in humans, as the polar TE mediate the contact with the endometrial epithelium. As these mural TE cells are the most distant from the ICM, the cell compartment that produces the signaling maintain the stemness of TE lineage, mural TE would undergo differentiation and entry into a specific type of cell cycle-endoreduplication, DNA replication without cytokinesis, to form trophoblast giant cells [24]. The analysis of cell proliferation 
status has revealed the polar TE actively proliferates after the termination of blastocyst dormancy, while the mural TE cells are still Ki67 negative. Consistent with this observation, the trophoblast stemness marker, $C d \times 2$, disappeared in the mural TE cells once the blastocyst was activated, meanwhile, differentiation markers, such as Plac1 are specifically upregulated in the mural TE cells [25]. This observation suggests the mural TE cells undergo a differentiation program to initiate their attachment during implantation. One of the cellular behaviors of trophoblast cells during the attachment is the invasion, accompanied with the extensive remodeling of tight junction and extracellular matrix. Electron microscope observation also confirmed these changes and demonstrated that they occur principally in the mural TE cells. The mural TE cell differentiation with loss of cell stemness and acquisition of invasion capacity was also found in the normal implantation between the day 4 morning and evening blastocyst in our study.

\section{Embryo-derived signals for dialogue with uterus during the attachment}

During the implantation, the cell communication between the blastocyst and uterus is needed to coordinate various cellular events. It was demonstrated that, before the attachment reaction on day 4 night, the blastocyst produces signals, such as HB-EGF, to crosstalk with the uterus and to induce uterine HB-EGF, expression on the afternoon of day 4 post coitum [26, 27]. Another study also found that lipid compounds in medium conditioned by culture with blastocysts can induced the activity of anandamide hydrolase (FAAH) in uterine cells [28]. Uterine anandamide levels are fine-tuned by FAAH and other enzymes, such as NAPE-PLD, and are critical for blastocyst activation $[29,30]$. Secretory and transmembrane proteins are main mediators of the dialog between the cells, and RNA-Seq revealed some secretory molecules were differentially expressed between the dormant and reactivated blastocysts, including the $\mathrm{Hb}$-egf. Wnt signaling has been shown to be indispensable for the blastocyst to acquire the implantation competency, and the RNA-Seq data also demonstrate that different members in this pathway were dynamically expressed during the reactivation process [31].

The proinflammatory regulators account for a large proportion of the differentially expressed genes. Implantation is considered an inflammatory-like attachment response, which is conserved among multiple species [32]. The mechanisms by which the inflammatory response are initiated during implantation still remain elusive [33]. The presence of these embryo-derived inflammatory molecules suggests the embryo could also be involved in this process. Affi-Gel beads soaked with recombinant protein is a simple model to mimic the blastocyst to explore the function of an embryo-derived signal [34]. One of these molecules, S100A9, a calcium binding protein, was found to induce the attachment-like blue band reaction, as well as the expression of COX2, an inflammatory factor essential for implantation in the stromal cells surrounding the beads. In the RNA-seq data, S100A9 increased after 6 hour of estrogen treatment, and another inflammatory factor, TNF $\alpha$, was upregulated immediately in the first six hours of estrogen treatment.

Furthermore, TNF $\alpha$ can induce the activation of NFKB signaling pathway expression of S100A9 in the blastocyst, consistent with the previous report in other cell types [35]. This TNF $\alpha-N F k B-S 100 A 9$ cascade reveals an autocrine manner of TNF $\alpha$ by which embryos produce an adequate inflammatory signal to the uterus. Indeed, in the 12 hours after the estrogen treatment, the mRNA of Tnfa is downregulated in the blastocyst. The protein can be still detected, suggesting that the inflammatory response during the attachment is precisely controlled at an appropriate level. Moreover, it was reported recently that $\mathrm{t}$ S100A9 has an autoinhibitory influence to locally restrict sterile inflammation [36]. This may give some explanation of why the S100A9 was utilized as an inflammatory signal during the attachment. Interestingly, a recent study disclosed that the chromatin for of genes related to the inflammatory response tends to open for a short time in the mouse and human embryo at later blastocyst stages when the attachment response occurs. This also may give some clue about the tightly regulated expression for these genes. 
Leukemia inhibitory factor (LIF) is a member of the interleukin 6 (IL6) family and is indispensable for blastocyst implantation, as LIF knockout female mice are infertile due to implantation failure [37]. In the RNA-seq data, the blastocyst-expressed lif was downregulated during reactivation [12]. LIF protein can replace estrogen in induction of the differentiation of uterus and successful implantation in the pseudopregnant delayed model [38]. Surprisingly, LIF alone failed to reactivate blastocyst implantation in the pregnant delayed model, although it can facilitate estrogen in inducing this process when combined with a suboptional dose of estrogen injection. This suggests that LIF signaling may not be a factor in blastocyst activation in the delayed model. The elevated expression of LIF in the dormant blastocyst suggested that embryonic LIF signaling may be involved in the maintenance of different cell lineage in blastocyst In support of this view, it has been demonstrated that epiblast in blastocyst from LIF coreceptor gp130 knockout embryos would undergo apoptosis in the delayed model [39].

\section{Conclusion}

Embryo implantation involves the synchronized interaction between an implantation-competent blastocyst and a receptive uterus. Genetic evidence has demonstrated a wide range of regulatory molecules as essential players in conferring uterine receptivity in mouse [4, 9]. Many of these molecules have also been confirmed to have evolutionarily conserved function in human endometrium. However, it remains largely unknown how blastocysts achieve implantation competency. Previous studies have explored the ultrastructure and cellular changes during the blastocyst in the delayed model, and recent molecular approaches, particularly the -omics studies, have provided more profound understanding of the molecular mechanisms of these cellular events during the blastocyst activation, including information on metabolism and cell cycle activity. Moreover, the RNA-seq data for genetic landscape of blastocysts for implantation in a diapause model revealed different cell differentiation programs for ICM and TE lineage. It was also found that the implantation-competent blastocysts produce proinflammatory signals to initiate the inflammatory-like response in the receptive endometrium. The -omics research during the blastocyst activation process has given some novel understanding for this critical event of pregnancy. These studies have used multiple embryos to conduct the experiment, and the information for different molecular changes in different lineage cells is limited, and the epigenetic regulation underlying these dynamic transcriptional changes is also unknown. The recent report on the single cell transcriptome and DNA methylome of in vitro cultured peri-implantation human embryos has provide an example of the advances in understanding the cell behavior of different embryonic lineages during the implantation based on the new technology [40]. The recently developed low-input genome-wide single cell transcriptional and epigenetic mark studies have provided powerful tools to explore these important questions [41, 42].

\section{Declaration of interest}

The authors declare no competing interests. 


\section{Funding}

This work was supported by the National Key R\&D Program of China (2017YFC1001402 to H.W., 2018 YFC1004401 to S.K.); the National Natural Science Foundation of China (81830045 to H.W., 81971388 to S.K.); Fundamental Research Funds for the Central Universities (20720180039 to S.K.).

\section{Acknowledgements}

Thanks all the lab members for their efforts in collecting the embryos; Dr. Wenbo Deng for thoughtful discussion and input.

\section{References}

[1] Wang $\mathrm{H}$ and Dey SK. 2006 Roadmap to embryo implantation: clues from mouse models. Nat Rev Genet. 7(3) 185-99.

[2] Zhang $\mathrm{S}$ et al. 2013 Physiological and molecular determinants of embryo implantation. Mol Aspects Med 34(5) 939-80.

[3] Smith MB and Paulson RJ. 2019 Endometrial preparation for third-party parenting and cryopreserved embryo transfer. Fertil Steril 111(4) 641-649.

[4] Cha J, Sun X and Dey SK. 2012 Mechanisms of implantation: strategies for successful pregnancy. Nat Med 18(12) 1754-67.

[5] Lim HJ and Wang H. 2010 Uterine disorders and pregnancy complications: insights from mouse models. J Clin Invest 120(4) 1004-15.

[6] Yoshinaga K and Adams CE. 1966 Delayed implantation in the spayed, progesterone treated adult mouse. J Reprod Fertil 12(3) 593-5.

[7] Paria BC, Huet-Hudson YM and Dey SK. 1993 Blastocyst's state of activity determines the "window" of implantation in the receptive mouse uterus. Proc Natl Acad Sci USA 90(21) 10159-62.

[8] Paria BC et al. 1998 Coordination of differential effects of primary estrogen and catecholestrogen on two distinct targets mediates embryo implantation in the mouse. Endocrinology 139(12) 5235-46.

[9] Kong S et al. 2019 Epigenetic control of embryo-uterine crosstalk at peri-implantation. Cell Mol Life Sci.

[10] Hamatani T et al. 2004 Global gene expression analysis identifies molecular pathways distinguishing blastocyst dormancy and activation. Proc Natl Acad Sci USA 101(28) 10326-31.

[11] Fu Z et al. 2014 Integral proteomic analysis of blastocysts reveals key molecular machinery governing embryonic diapause and reactivation for implantation in mice. Biol Reprod 90(3) 52.

[12] He B et al. 2019 Blastocyst activation engenders transcriptome reprogram affecting X-chromosome reactivation and inflammatory trigger of implantation. Proc Natl Acad Sci USA 116(33) 16621-16630.

[13] Fu Z et al. 2013 Molecular and cellular aspects of blastocyst dormancy and reactivation for implantation. J Stem Cells 8(2) 59-77.

[14] Bulut-Karslioglu A et al. 2016 Inhibition of mTOR induces a paused pluripotent state. Nature 540(7631) 119-123.

[15] Scognamiglio R et al. 2016 Myc Depletion Induces a Pluripotent Dormant State Mimicking Diapause. Cell 164(4) 668-80.

[16] Lee JE et al. 2011 Autophagy regulates embryonic survival during delayed implantation. Endocrinology 152(5) 2067-75.

[17] Shin $\mathrm{H}$ et al. 2017 The formation of multivesicular bodies in activated blastocysts is influenced by autophagy and FGF signaling in mice. Sci Rep 741986.

[18] Lefevre PL, Palin MF and Murphy BD. 2011 Polyamines on the reproductive landscape. Endocr Rev 32(5) 694-712. 
[19] Fenelon JC and Murphy BD. 2017 Inhibition of polyamine synthesis causes entry of the mouse blastocyst into embryonic diapause. Biol Reprod 97(1) 119-132.

[20] Zhang $\mathrm{H}$ et al. 2019 Polyamines Control elF5A Hypusination, TFEB Translation, and Autophagy to Reverse B Cell Senescence. Mol Cell.

[21] Evans MJ and Kaufman MH. 1981 Establishment in culture of pluripotential cells from mouse embryos. Nature 292(5819) 154-6.

[22] Borensztein $\mathrm{M}$ et al. 2017 Contribution of epigenetic landscapes and transcription factors to Xchromosome reactivation in the inner cell mass. Nat Commun 8(1) 1297.

[23] Sahakyan A, Yang Y and Plath K. 2018 The Role of Xist in X-Chromosome Dosage Compensation. Trends Cell Biol 28(12) 999-1013.

[24] Rossant J and Cross JC. 2001 Placental development: lessons from mouse mutants. Nat Rev Genet 2(7) 538-48.

[25] Chang WL et al. 2014 Role of placenta-specific protein 1 in trophoblast invasion and migration. Reproduction 148(4) 343-52.

[26] Lim HJ and Dey SK. 2009 HB-EGF: a unique mediator of embryo-uterine interactions during implantation. Exp Cell Res 315(4) 619-26.

[27] Das SK et al. 1994 Heparin-binding EGF-like growth factor gene is induced in the mouse uterus temporally by the blastocyst solely at the site of its apposition: a possible ligand for interaction with blastocyst EGF-receptor in implantation. Development 120(5) 1071-83.

[28] Maccarrone $M$ et al. 2004 Mouse blastocysts release a lipid which activates anandamide hydrolase in intact uterus. Mol Hum Reprod 10(4) 215-21.

[29] Guo Y et al. $2005 \mathrm{~N}$-acylphosphatidylethanolamine-hydrolyzing phospholipase D is an important determinant of uterine anandamide levels during implantation. J Biol Chem 280(25) 23429-32.

[30] Wang H et al. 2003 Differential G protein-coupled cannabinoid receptor signaling by anandamide directs blastocyst activation for implantation. Proc Natl Acad Sci USA 100(25) 14914-9.

[31] Xie H et al. 2008 Inactivation of nuclear Wnt-beta-catenin signaling limits blastocyst competency for implantation. Development 135(4) 717-27.

[32] Griffith OW et al. 2017 Embryo implantation evolved from an ancestral inflammatory attachment reaction. Proc Natl Acad Sci USA 114(32) E6566-E6575.

[33] Liu JL. 2018 Implantation in eutherians: Which came first, the inflammatory reaction or attachment? Proc Natl Acad Sci USA 115(1) E1-E2.

[34] Paria BC et al. 2001 Cellular and molecular responses of the uterus to embryo implantation can be elicited by locally applied growth factors. Proc Natl Acad Sci USA 98(3) 1047-52.

[35] Xu K and Geczy CL. 2000 IFN-gamma and TNF regulate macrophage expression of the chemotactic S100 protein S100A8. J Immunol 164(9) 4916-23.

[36] Vogl T et al. 2018 Autoinhibitory regulation of S100A8/S100A9 alarmin activity locally restricts sterile inflammation. J Clin Invest 128(5) 1852-1866.

[37] Stewart CL et al. 1992 Blastocyst implantation depends on maternal expression of leukaemia inhibitory factor. Nature 359(6390) 76-9.

[38] Chen JR, et al. 2000 Leukemia inhibitory factor can substitute for nidatory estrogen and is essential to inducing a receptive uterus for implantation but is not essential for subsequent embryogenesis. Endocrinology 141(12) 4365-72.

[39] Nichols J et al. 2001 Physiological rationale for responsiveness of mouse embryonic stem cells to gp130 cytokines. Development 128(12) 2333-9.

[40] Zhou F et al. 2019 Reconstituting the transcriptome and DNA methylome landscapes of human implantation. Nature 572(7771) 660-664.

[41] Kaya-Okur HS et al. 2019 CUT\&Tag for efficient epigenomic profiling of small samples and single cells. Nat Commun 10(1) 1930.

[42] Ai S et al. 2019 Profiling chromatin states using single-cell itChIP-seq. Nat Cell Biol 21(9) 1164-1172. 


\section{Audience Discussion}

Marilyn Renfree: You show differences in the time of activation of polar versus mural trophectoderm in your study. Can you speculate about the signaling mechanisms by which this might be regulated?

Shuangbo Kong: In our study, we mainly focused on the cellular differences between polar versus mural trophectoderm, and we don't have the data for the time of activation these two cell compartments at present. In mice, it's mainly the mural trophectoderm which contacts with the uterine epithelium, mediating the attachment reaction during implantation. The mural trophectoderm will differentiate as it ceases cell division and then initiates endoreduplication to form the primary trophoblast giant cells after the attachment reaction. One reason for the difference between the polar versus mural trophectoderm of blastocyst is whether they are located near or distant from the inner cell mass (ICM), which can produce the signal, such as Fgf4 to maintain the stemness of trophoblast. The mural trophectoderm, which is far from the ICM, and may not receive the sufficient self-renewal signaling, would undergo the default differentiation pathway which had been demonstrated in the trophoblast stem cells, such as the loss of stemness marker expression and inductions of cell differentiation markers. So, this intrinsic difference between the polar versus mural trophectoderm may determine the different cellular behavior during the attachment. The polar trophectoderm needs to maintain the high rate of proliferation and self-renewal ability to assure postimplantation extraembryonic ectoderm morphogenesis. Meanwhile, the mural trophectoderm undergoes the differentiation program, including the ability to clear the uterine epithelium during invasion. Furthermore, we can also not exclude the possibility that the uterine signaling may be also involved for the differences between the polar versus mural trophectoderm, since the mural trophectoderm is in closer contact with the epithelium during the attachment.

Bruce Murphy: How do the inflammatory models produced by the embryo affect the endometrium? Is it possible to terminate diapause using inflammatory cytokines?

Shuangbo Kong: In our data, we find several inflammatory molecules, including the Tnfa, Saa3, S100A8/S100A9, were increased during the blastocyst activation, and when beads coated with the S100A9 were transferred into pseudopregnant uteri, the attachment-like blue reaction was induced around the transferred beads. This suggested the inflammatory molecules such as the S100a9 produced by the blastocyst can mediate the cross-talk between the embryo and the uterus. The TLR4 receptor for S100A9 was also expressed in the uterine epithelium, which suggested the embryo derived S100A9 may bind with the epithelial receptor to induce the down-stream inflammatory response in the attachment reaction, such as COX2 induction. Besides the inflammatory response in the uterine compartment during implantation, we also propose there is a precisely controlled inflammatory response in the embryo, since we find the transient early induction of Tnfa during the transition of blastocyst from dormancy to the activated state, and this Tnfa may function in an autocrine manner to induce expression of another inflammatory mediator, S100A9 in embryos. It is not clear why the embryos switch the expression of inflammatory mediator during the early ( $6 \mathrm{~h}$ after the estrogen) and later ( $12 \mathrm{~h}$ after the estrogen) time of activation. Since the S100A9 was reported to have an autoinhibitory influence to locally restrict sterile inflammation, employing S100A9 as the mediator between the embryo and uterus may be beneficial for the controlled inflammatory response during implantation. With respect to the signal to initiate the inflammatory response in the embryo, we have a hypothesis that the uterus may first produce a weak inflammatory signal along its length in response to the estrogen treatment in the delayed model. While the implantation chamber with a blastocyst amplifies this inflammatory response in response and form a positive amplification loop to induce sufficient inflammatory mediators to induce the attachment reaction. The $M s \times 1 / 2$ deleted uteri display sustained inflammation and inability to maintain blastocyst dormancy, as the blastocysts in Msx1/2 deleted uteri can induce the implantation-like blue band responses in the delayed model without estrogen treatment. This suggests that precisely controlled inflammation status in the uterus is critical for implantation. In support of this view, it is known that metabolic activation of dormant blastocysts in vitro is not sufficient for the embryo implantation, whether the combination of inflammatory cytokine treatment and metabolism activation could endow the implantation competency of blastocyst is an interesting concern and merits the further study. 